\title{
TERT Promoter Mutations and Tumor Persistence/Recurrence in Papillary Thyroid Cancer
}

\author{
Jae Kyung Myung, MD \\ Byung Kuk Kwak, MS² \\ Jung Ah Lim, MD, PhD ${ }^{3}$ \\ Myung-Chul Lee, MD, $\mathrm{PhD}^{4}$ \\ Min Joo Kim, MD, PhD²
}

Departments of ${ }^{1}$ Pathology and

${ }^{2}$ Internal Medicine,

Korea Cancer Center Hospital, Seoul,

${ }^{3}$ Department of Internal Medicine,

National Medical Center, Seoul,

${ }^{4}$ Department of Otorhinolaryngology,

Korea Cancer Center Hospital, Seoul, Korea

\section{Purpose}

A telomerase reverse transcriptase (TERT) promoter mutation was identified in thyroid cancer. This TERT promoter mutation is thought to be a prognostic molecular marker, because its association with tumor aggressiveness, persistence/recurrence, and disease-specific mortality in papillary thyroid carcinoma (PTC) has been reported. In this study, we attempted to determine whether the impact of the TERT promoter mutation on PTC persistence/ recurrence is independent of clinicopathological parameters.

\section{Materials and Methods}

Using propensity score matching, 39 patients with PTC persistence or recurrence were matched with 35 patients without persistence or recurrence, with a similar age, sex, tumor size, multifocality, bilaterality, extrathyroidal extension, and lymph node metastasis. The TERT promoter and the BRAF V600E mutations were identified from PTC samples.

\section{Results}

The TERT promoter mutation was detected in $18 \%$ of PTC patients (13/74). No significant difference in the frequency of the TERT promoter mutation was observed between the persistence/recurrence group and the non-recurrence group.

\section{Conclusion}

These results suggest that the prognostic implications of the TERT promoter mutation are dependent on clinicopathological parameters.
Correspondence: Min Joo Kim, MD, PhD Department of Internal Medicine, Korea Cancer Center Hospital, 75, Nowon-ro, Nowon-gu, Seoul 01812, Korea

Tel: 82-2-970-1215

Fax: 82-2-970-2438

E-mail: chorong24@gmail.com
Key words

Papillary thyroid cancer, Telomerase, Thyroid neoplasms

\section{Introduction}

In cancer cells, maintenance of telomere length plays an important role in cellular stability and immortality [1]. Telomerase reverse transcriptase (TERT) is the catalytic subunit of telomerase, and the telomerase synthesizes repetitive DNA sequences for maintenance of telomere length [1]. Telomerase activation has been reported in several cancers including melanoma, breast cancer, and head and neck can- cer [2-4].

Telomerase activation has also been identified in thyroid cancers [5]. Approximately $66 \%$ of all thyroid cancers exhibit telomerase activation [5]. However, no telomerase activation has been observed in normal thyroid tissues, suggesting that it is cancer-specific [6,7]. Therefore, telomerase activation has a potential for use as a marker of thyroid cancer.

Telomerase activation can be induced by a TERT promoter mutation [4]. Two hot spots of TERT promoter mutation in thyroid cancer cells have recently been reported $[124 \mathrm{G}>\mathrm{A}$ 
(C228T), 146G $>$ A (C250T)] [8-10]. The frequency of mutation differs according to the histological type of thyroid cancer. The TERT promoter mutation has been detected in 7\%-22\% of papillary thyroid carcinomas (PTCs) [9-11] and 11\%-25\% of follicular thyroid carcinomas $[8,11,12]$. However, this mutation was significantly more frequent in poorly differentiated carcinomas or anaplastic carcinomas (29\%-50\%) $[8,11,12]$.

Association of the TERT promoter mutation with poor prognosis of thyroid cancer has been reported. The TERT promoter mutation was associated with older age, larger tumor size, higher stage, and distant metastasis [10-12], and with tumor persistence / recurrence and disease-specific mortality [11-13]. However, whether the association with an increased risk of tumor persistence/recurrence is independent of clinicopathological parameters remains unknown. In the current study, to assess the possible importance of the TERT promoter mutation on tumor persistence/recurrence, the TERT promoter mutation in persistence/recurrence group of patients was compared with a matched non-recurrence control group of patients.

\section{Materials and Methods}

\section{Patients}

This study included patients who underwent total thyroidectomy at the Korea Cancer Center Hospital between 2006 and 2012, and had PTC surgical specimens of $1.0 \mathrm{~cm}$ or larger. The persistence/recurrence group was selected among patients with tumor recurrence or persistence followed by a second surgery in our hospital. Persistence/ recurrence was defined as the presence of a structural abnormality confirmed by surgical pathology. The non-recurrence group was defined as patients without evidence of disease or recurrence for more than 5 years after surgery. The study was approved by the Institutional Review Board of the Korea Cancer Center Hospital (K-1501-002-036), and followed the tenets of the Declaration of Helsinki.

Electronic medical records were reviewed retrospectively for collection of data on patient demographics and tumor stage, and surgical specimens were obtained for genetic analyses.

\section{DNA extraction}

Formalin-fixed, paraffin-embedded PTC tissues were cut into $10 \mu \mathrm{m}$ sections. DNA was extracted using the QIAmp DNA FFPE Tissue Kit (Qiagen, Hilden, Germany) following the manufacturer's instructions.

\section{TERT promoter mutation}

Polymerase chain reaction (PCR) was performed, followed by Big Dye sequencing for identification of the TERT promoter mutation. A 235-bp fragment containing the TERT promoter mutations $\mathrm{C} 228 \mathrm{~T}$ and $\mathrm{C} 250 \mathrm{~T}$ on the genomic DNA was amplified by PCR. The quality of the PCR product was confirmed by gel electrophoresis, and its sequencing was performed using a Big Dye terminator v3.1 Cycle Sequencing Kit (Applied Biosystems, Foster City, CA) and an ABI PRISM 3130xl Genetic Analyzer (Applied Biosystems). When a mutation was found by Big Dye sequencing using the sense primer, the reaction was validated using the antisense primer.

\section{BRAF V600E mutation}

Real-time PCR was performed using the TaqMan MGB probes and FastStart Taq DNA Polymerase (Roche Life Science, Indianapolis, IN) for identification of the BRAF V600E mutation. Amplification and detection were performed using an ABI prism 7500 Sequence Detection System (Applied Biosystems).

\section{Statistical analysis}

Based on a previous study [13], we hypothesized that the frequency of the TERT promoter mutation was detectable in $7.5 \%$ of the non-recurrence group and $36.3 \%$ of the persistence/recurrence group, and the sample size of the study was calculated with a type 1 error $(\alpha)$ of 0.05 and a power $(\beta)$ of $80 \%$, resulting in 32 patients in each group.

Propensity score matching was performed to adjust for differences in the other clinicopathological features that could confound tumor persistence/recurrence. The propensity score was calculated using logistic regression with the age, sex, tumor size, multifocality, bilaterality, extrathyroidal extension (ETE), and lymph node metastasis. Thirty-nine patients with tumor persistence or recurrence were identified and 39 patients without tumor recurrence were matched using the propensity scores. Among the 39 patients from the non-recurrence group, four patients were excluded because of missing formalin-fixed, paraffin-embedded tissues or poor DNA quality, and only 35 patients were included in this study. Finally, 74 patients were analyzed. The frequencies of the TERT promoter and the BRAF V600E mutations were compared between the persistence/ recurrence group and the non-recurrence group. Data are expressed as a percentage or mean \pm standard deviation. The independent $t$ test or chisquare test was used when appropriate. A p-value $<0.05$ was 
Table 1. Clinicopathological characteristics of patients

\begin{tabular}{|c|c|c|c|}
\hline Characterisic & Persistence/Recurrence group (n=39) & Non-recurrence group ( $\mathrm{n}=35$ ) & p-value \\
\hline Age (yr) & $49 \pm 13$ & $47 \pm 14$ & 0.64 \\
\hline Women & $30(77)$ & $27(77)$ & $>0.99$ \\
\hline Tumor size (cm) & $2.6 \pm 1.6$ & $2.6 \pm 1.3$ & 0.94 \\
\hline Multifocality & $25(64)$ & $27(77)$ & 0.31 \\
\hline Bilaterality & $20(51)$ & $21(60)$ & 0.36 \\
\hline ETE & $35(90)$ & $31(90)$ & $>0.99$ \\
\hline \multicolumn{4}{|l|}{ LN metastasis } \\
\hline Central LN & $17(44)$ & $14(40)$ & 0.82 \\
\hline Lateral LN & $22(56)$ & $21(60)$ & \\
\hline \multicolumn{4}{|l|}{ Tumor stage } \\
\hline I-II & $16(41)$ & $16(46)$ & 0.82 \\
\hline III-IV & $23(59)$ & $19(54)$ & \\
\hline RAI & $37(95)$ & $33(94)$ & $>0.99$ \\
\hline RAI dose (mCi) & $208 \pm 105$ & $199 \pm 125$ & 0.72 \\
\hline
\end{tabular}

Values are presented as mean \pm standard deviation or number (\%). ETE, extrathyroidal extension; LN, lymph node; RAI, radioactive iodine; $\mathrm{mCi}$, millicurie.

considered statistically significant. Statistical analyses were performed using SPSS ver. 22.0 (IBM Co., Armonk, NY).

\section{Results}

The analyses included 39 patients from the persistence/ recurrence group and 35 matched patients from the nonrecurrence group, using propensity scores based on age, sex, tumor size, multifocality, bilaterality, ETE, and lymph node metastasis. Patient demographics and pathological findings of surgical specimens are summarized in Table 1 . The mean age of patients was $48 \pm 14$ years, and $77 \%$ of the patients were female. The mean size of the primary tumor was $2.6 \pm 1.4 \mathrm{~cm}$. Most patients (90\%) had ETE and all patients had lymph node metastasis. No patient had distant metastasis. There were no significant differences in clinicopathological characteristics between patients from the persistence/recurrence and non-recurrence groups. Among the persistence/recurrence group, 25 patients had persistent disease and 14 recurred. Neck lymph nodes were the most common site of disease persistence/recurrence.

The time interval from the initial surgery to the identification of persistence/ recurrence was $1.8 \pm 1.2$ years in the persistence/recurrence group and the median follow-up time was $7.5 \pm 1.0$ years in the non-recurrence group.

The TERT promoter mutation was detected in $18 \%$ of PTC samples (13/74). The frequency of the TERT promoter mutation was slightly higher in the persistence/recurrence group than in the non-recurrence group ( $23 \%$ vs. $12 \%$, respectively), but there was no statistical significance $(\mathrm{p}=0.23)$ (Table 2). Most of the mutations (85\%) presented the C228T mutation and the others presented the $\mathrm{C} 250 \mathrm{~T}$ mutation. The overall BRAF V600E mutation was observed in $61 \%$ of PTC samples (45/74), comprised of $54 \%$ in the persistence/ recurrence group and $69 \%$ in the non-recurrence group (Table 2). No statistical difference in the BRAF V600E mutation was observed between patients in the persistence/recurrence and non-recurrence groups $(\mathrm{p}=0.24)$.

The patients were then divided according to their TERT promoter mutation status. As previously reported, the TERT promoter mutation is associated with several clinicopathological parameters (Table 3). Patients with the TERT promoter mutation were significantly older, had larger tumor sizes, and higher tumor stages compared to patients with the wild-type TERT promoter.

\section{Discussion}

In the current study, we showed that the TERT promoter mutation in patients from the persistence/recurrence group was not different from patients in the non-recurrence group who had similar clinicopathological characteristics. The results suggest that the TERT promoter mutation has no additional effect on the tumor persistence/recurrence of 
Table 2. TERT promoter and BRAF V600E mutations

\begin{tabular}{ccccc} 
Mutation & Total & Persistence/Recurrence group $(\mathbf{n}=39)$ & Non-recurrence group $(\mathbf{n}=35)$ & p-value \\
TERT mutation & $13(18)$ & $9(23)$ & $4(12)$ & 0.23 \\
C228T & $11(15)$ & $8(20)$ & $3(9)$ & $1(3)$ \\
C250T & $2(3)$ & $1(3)$ & $24(69)$ & 0.24 \\
\hline BRAF mutation & $45(61)$ & $21(54)$ & \\
\hline
\end{tabular}

Values are presented as number (\%). TERT, telomerase reverse transcriptase.

Table 3. TERT promoter mutation and clinicopathological parameters

\begin{tabular}{|c|c|c|c|}
\hline Parameter & Mutated TERT $(\mathrm{n}=13)$ & Wild-type TERT $(\mathrm{n}=61)$ & p-value \\
\hline Age (yr) & $61 \pm 10$ & $45 \pm 13$ & $<0.01$ \\
\hline Women & $11(85)$ & $46(75)$ & 0.72 \\
\hline Tumor size $(\mathrm{cm})$ & $3.7 \pm 2.0$ & $2.4 \pm 1.2$ & 0.03 \\
\hline Multifocality & $6(46)$ & $46(75)$ & 0.04 \\
\hline Bilaterality & $4(31)$ & $36(59)$ & 0.07 \\
\hline ETE & $13(100)$ & $53(87)$ & 0.34 \\
\hline \multicolumn{4}{|l|}{ LN metastasis } \\
\hline Central LN & $7(54)$ & $24(39)$ & 0.37 \\
\hline Lateral LN & $6(46)$ & $37(61)$ & \\
\hline \multicolumn{4}{|l|}{ Tumor stage } \\
\hline I-II & $1(8)$ & $31(51)$ & $<0.01$ \\
\hline III-IV & $12(92)$ & $30(49)$ & \\
\hline Duration of follow-up & $3.4 \pm 3.4$ & $4.7 \pm 3.0$ & 0.17 \\
\hline Persistence/Recurrence & $9(69)$ & $30(49)$ & 0.23 \\
\hline
\end{tabular}

Values are presented as mean \pm standard deviation or number $(\%)$. TERT, telomerase reverse transcriptase; ETE, extrathyroidal extension; LN, lymph node.

PTC.

Association of the TERT promoter mutation with tumor persistence/ recurrence and the disease-specific mortality of PTC has been reported [11-13]. However, it remains uncertain whether its association is independent of clinicopathological parameters. In the current study, in comparison of patients with similar clinicopathological parameters, the TERT promoter mutation did not show a significant association with tumor persistence/recurrence. Xing et al. [13] reported significant association of the TERT promoter mutation with tumor recurrence, but there was no significance after adjustment for age, sex, multifocality, tumor size, ETE, vascular invasion, and lymph node metastasis. Although the relationship between the TERT promoter mutation and disease-specific mortality was significant after adjustment for age and sex [11,12], multivariate analysis for tumor size or lymph node metastasis has never been performed. Therefore, for definitive assessment of the possible impact of the TERT promoter mutation on the prognosis of PTC, it should be confirmed in larger studies like the BRAF mutation $[14,15]$.
The BRAF V600E mutation also showed an association with tumor recurrence and mortality of PTC, but its association was no longer significant after the adjustment of clinicopathological parameters [14]. These results suggested that the prognostic value of the BRAF mutation was dependent on clinicopathological parameters.

The TERT promoter mutation has been reported in 7\%$22 \%$ of PTC cases [9-11] and in 11\% of Chinese patients with PTC [10]. The frequency of the TERT promoter mutation $(18 \%)$ was also similar in this study. However, because the current study excluded PTC samples smaller than $1.0 \mathrm{~cm}$, the overall frequency of the TERT promoter mutation in Korea or Asia may be lower than in Western countries.

Telomere shortening is closely associated with both cancer and aging [16]. Telomere length shortens with age [17], and a shortened telomere can activate telomerase and the TERT promoter mutation [12]. Consistent with our results in the current study, previous studies reported a consistent relationship between the TERT promoter mutation and age [1012]. In addition, the TERT promoter mutation was found 
more frequently in larger tumors, and there were little or no mutations in papillary thyroid microcarcinomas $[10,11,18]$. These results suggest that vigorous proliferation of cancer cells triggers the TERT promoter mutation or telomerase activation.

Past studies have reported an association of the TERT promoter and BRAF mutations [11,13]. The BRAF mutation is a well-known prognostic molecular marker [19], and PTCs with BRAF V600E mutations showed more aggressive clinicopathological characteristics $[20,21]$. In addition, patients with both the TERT C228T and BRAF V600E mutations were associated with higher risk clinicopathological characteristics or tumor recurrence, suggesting a synergistic effect between the two mutations [13]. However, no association was found in another study [11]. In this study, their association could not be determined because of small sample size.

\section{Conclusion}

In summary, the frequency of the TERT promoter mutation did not differ between patients with similar clinicopathological characteristics in the persistence/recurrence and non-recurrence groups, suggesting that the prognostic implications of the TERT promoter mutation were dependent on clinicopathological parameters.

\section{Conflicts of Interest}

Samkwang Medical Laboratories helped in identification of mutation.

\section{Acknowledgments}

This study was supported by a grant from the RTR (Radiological Translational Research program), Korea Institute of Radiological \& Medical Sciences (50472-2015).

\section{References}

1. Smekalova EM, Shubernetskaya OS, Zvereva MI, Gromenko EV, Rubtsova MP, Dontsova OA. Telomerase RNA biosynthesis and processing. Biochemistry (Mosc). 2012;77:1120-8.

2. Kim NW, Piatyszek MA, Prowse KR, Harley CB, West MD, Ho PL, et al. Specific association of human telomerase activity with immortal cells and cancer. Science. 1994;266:2011-5.

3. Clark GM, Osborne CK, Levitt D, Wu F, Kim NW. Telomerase activity and survival of patients with node-positive breast cancer. J Natl Cancer Inst. 1997;89:1874-81.

4. Huang FW, Hodis E, Xu MJ, Kryukov GV, Chin L, Garraway LA. Highly recurrent TERT promoter mutations in human melanoma. Science. 2013;339:957-9.

5. Soares P, Lima J, Preto A, Castro P, Vinagre J, Celestino R, et al. Genetic alterations in poorly differentiated and undifferentiated thyroid carcinomas. Curr Genomics. 2011;12:609-17.

6. Matthews P, Jones CJ, Skinner J, Haughton M, de Micco C, Wynford-Thomas D. Telomerase activity and telomere length in thyroid neoplasia: biological and clinical implications. J Pathol. 2001;194:183-93.

7. Capezzone M, Cantara S, Marchisotta S, Busonero G, Formichi
$\mathrm{C}$, Benigni $\mathrm{M}$, et al. Telomere length in neoplastic and nonneoplastic tissues of patients with familial and sporadic papillary thyroid cancer. J Clin Endocrinol Metab. 2011;96:E1852-6.

8. Liu X, Bishop J, Shan Y, Pai S, Liu D, Murugan AK, et al. Highly prevalent TERT promoter mutations in aggressive thyroid cancers. Endocr Relat Cancer. 2013;20:603-10.

9. Landa I, Ganly I, Chan TA, Mitsutake N, Matsuse M, Ibrahimpasic $\mathrm{T}$, et al. Frequent somatic TERT promoter mutations in thyroid cancer: higher prevalence in advanced forms of the disease. J Clin Endocrinol Metab. 2013;98:E1562-6.

10. Liu X, Qu S, Liu R, Sheng C, Shi X, Zhu G, et al. TERT promoter mutations and their association with BRAF V600E mutation and aggressive clinicopathological characteristics of thyroid cancer. J Clin Endocrinol Metab. 2014;99:E1130-6.

11. Melo M, da Rocha AG, Vinagre J, Batista R, Peixoto J, Tavares $\mathrm{C}$, et al. TERT promoter mutations are a major indicator of poor outcome in differentiated thyroid carcinomas. J Clin Endocrinol Metab. 2014;99:E754-65.

12. Liu T, Wang N, Cao J, Sofiadis A, Dinets A, Zedenius J, et al. The age- and shorter telomere-dependent TERT promoter 
mutation in follicular thyroid cell-derived carcinomas. Oncogene. 2014;33:4978-84.

13. Xing M, Liu R, Liu X, Murugan AK, Zhu G, Zeiger MA, et al. BRAF V600E and TERT promoter mutations cooperatively identify the most aggressive papillary thyroid cancer with highest recurrence. J Clin Oncol. 2014;32:2718-26.

14. Xing M, Alzahrani AS, Carson KA, Viola D, Elisei R, Bendlova $\mathrm{B}$, et al. Association between BRAF V600E mutation and mortality in patients with papillary thyroid cancer. JAMA. 2013; 309:1493-501.

15. Xing M, Alzahrani AS, Carson KA, Shong YK, Kim TY, Viola $\mathrm{D}$, et al. Association between BRAF V600E mutation and recurrence of papillary thyroid cancer. J Clin Oncol. 2015;33: 42-50.

16. Blasco MA. Telomere length, stem cells and aging. Nat Chem Biol. 2007;3:640-9.

17. Canela A, Vera E, Klatt P, Blasco MA. High-throughput telom- ere length quantification by FISH and its application to human population studies. Proc Natl Acad Sci U S A. 2007;104: 5300-5.

18. de Biase D, Gandolfi G, Ragazzi M, Eszlinger M, Sancisi V, Gugnoni M, et al. TERT promoter mutations in papillary thyroid microcarcinomas. Thyroid. 2015;25:1013-9.

19. Xing M. BRAF mutation in thyroid cancer. Endocr Relat Cancer. 2005;12:245-62.

20. Xing M, Westra WH, Tufano RP, Cohen Y, Rosenbaum E, Rhoden KJ, et al. BRAF mutation predicts a poorer clinical prognosis for papillary thyroid cancer. J Clin Endocrinol Metab. 2005;90:6373-9.

21. Kim TH, Park YJ, Lim JA, Ahn HY, Lee EK, Lee YJ, et al. The association of the BRAF(V600E) mutation with prognostic factors and poor clinical outcome in papillary thyroid cancer: a meta-analysis. Cancer. 2012;118:1764-73. 